\title{
FLÜCHTLINGSKINDER
}

\section{Teilhabe ohne Vorbehalt}

\section{VON KATHARINA VOGT}

Katharina Vogt ist Referentin für Flüchtlingssozialarbeit beim Arbeiterwohlfahrt Bundesverband e. V. und Mitglied des Vorstands des Flüchtlingsrats Berlin.

E-Mail katharina.vogt@awo.org
Flüchtlingskinder, die die schwierigen Umstände einer Flucht mit und ohne Eltern gemeistert haben, verfügen aufgrund ihrer Erfahrungen und der Erwartungen der Zurückgebliebenen oft über erhebliche Ressourcen für den Bildungsprozess wie Durchsetzungsfähigkeit, Flexibilität, Entscheidungsbereitschaft, Ehrgeiz. Der Schlüssel zum Einsatz dieser Ressourcen ist jedoch die Möglichkeit zur Teilhabe.

Rund 8.000 Kinder warten hierzulande auf ihre Entscheidung im Asylverfahren und etwa 30.000 Minderjährige leben in Deutschland lediglich »geduldet«. Im Jahr 2009 haben knapp 1.300 unbegleitete minderjährige Flüchtlinge einen Asylantrag gestellt. Fachkreise schätzen, dass bis zu 6.000 Kinder als unbegleitete, minderjährige Flüchtlinge ohne Eltern in Deutschland leben.

Im folgenden Beitrag wird die Situation von Flüchtlingskindern in Deutschland beschrieben. Unter dem Begriff »Flüchtlingskinder « versteht die Verfasserin sowohl Kinder, die im Rahmen eines Asylverfahrens um Schutz nachsuchen als auch Kinder, die als De-facto-Flüchtlinge nicht abgeschoben werden und in Deutschland leben. Auf Grundlage der UN-Kinderrechtskonvention beziehen sich die Ausführungen auf alle Kinder unter 18 Jahren und auf (von Eltern oder Verwandten) begleitete und unbegleitete minderjährige Flüchtlinge. Sie sind in allen Einrichtungen der sozialen Organisationen vereinzelt vertreten und werden insbesondere in den Erstaufnahmen für unbegleitete Minderjährige als auch in Wohnheimen für Flüchtlingsfamilien betreut.

Flüchtlingskinder und Kinder mit Duldung sind aufgrund eines fehlenden eindeutigen Aufenthaltsrechtes in Deutschland immer noch ausgegrenzt und ihnen werden Menschenrechte auf Bildung, gesundheitliche Versorgung sowie Teilhabe am gesellschaftlichen Leben verweigert:

- Dem Menschenrecht auf Bildung wird in Deutschland zwar in den letzten Jahren zunehmend Beachtung geschenkt; trotzdem ist die immer noch bestehende Unfähigkeit des deutschen Schulsystems, der sozialen Herkunft ausreichend Rechnung zu tragen, nicht zu verleugnen und trifft Flüchtlingskinder in mehrfacher Hinsicht.

- Flüchtlingskinder erhalten in Deutschland zwar den notwendigen Lebensunterhalt, aber entweder nur Sachleistungen oder aber einen Betrag in bar, der 35 Prozent unter dem Regelsatz nach SGB XII liegt, die gesundheitliche Versorgung ist auf ein Mindestmaß beschränkt.

- Flüchtlingskinder geraten in Deutschland immer wieder mit oder ohne Eltern in Abschiebehaft.

- Ab 16 Jahren sind sie eigenständig für die korrekte Durchführung eines überaus komplizierten rechtlichen Asylverfahrens verantwortlich, weil sie ausländer- und asylrechtlich bereits verfahrensfähig sind. Ein Verfahren, das so manchen deutschen Erwachsenen überfordern würde.

Im Jahre 1949 formulierten die Vereinten Nationen die »Erklärung der allgemeinen Menschenrechte « und entwickelten danach Übereinkommen für einzelne Zielgruppen. Auf dieser Grundlage wurde 1989 die UN-Kinderrechtskonvention verabschiedet. Sie bezieht sich nicht nur auf deutsche Kinder, sondern auch auf Kinder ohne deutschen Pass, die sich im Hoheitsgebiet tatsächlich aufhalten. Artikel 22 definiert zusätzlich die Kinder, die den Status als Flüchtling begehren, als besonders schutzwürdig.

Die Ungleichbehandlung von Flüchtlingskindern beruht auf dem sogenannten Vorbehalt der deutschen Bundesregierung: Die Bundesregierung ratifizierte damals die UN-Kinderrechtskonvention nur unter Vorbehalt des Fortbestehens bestehender Einschränkungen der Kinderrechte durch das Familien-und Ausländerrecht. Von Bedeutung für Flüchtlingskinder ist heute nur noch Punkt 4 der Erklärung: »Nichts in dem Übereinkommen kann dahin ausgelegt werden, dass die widerrechtliche Einreise eines Ausländers in das Gebiet der Bundesrepublik Deutschland oder dessen widerrechtlicher Aufenthalt dort erlaubt ist; auch kann keine Bestimmung dahin ausgelegt werden, dass sie das Recht der BRD be- 
schränkt, Gesetze und Verordnungen über die Einreise von Ausländern und die Bedingungen ihres Aufenthaltes zu erlassen oder Unterschiede zwischen Inländern und Ausländern zu machen. «

Die Wohlfahrtsverbände setzen sich seit vielen Jahren für die Rücknahme dieses Vorbehalts ein und nach Ansicht angesehener juristischer Gutachter ist der Vorbehalt rechtswidrig und daher sogar nichtig. Der Deutsche Bundestag sowie verschiedene Bundestagsausschüsse haben in den vergangenen 20 Jahren mehrfach die Rücknahme beschlossen. Auch die Koalitionsvereinbarung der neuen Bundesregierung enthält erneut die Forderung nach der Rücknahme. Auf die tatsächliche Umsetzung warten die Betroffenen bis heute.

\section{Die Rolle der Verbände}

Neben dem menschenrechtlichen Ansatz sind für Nichtregierungsorganisationen fachliche Standards der Jugendhilfe und jeweils innerverbandliche Grundsätze maßgeblich für ihre Arbeit. Soziales gesellschaftliches Handeln misst sich an Werten wie Solidarität, Toleranz, Freiheit, Gleichheit und Gerechtigkeit. Gerechtigkeit gründet in der gleichen Würde aller Menschen. Sie verlangt gleiche Rechte vor dem Gesetz und gleiche Chancen auf Teilhabe aber auch auf Ausgleich im Zugang zu Bildung, Ausbildung und Kultur.

Weltweit ist in den vergangenen Jahren durchaus viel für das Wohl von Kindern erreicht worden. Auch in der Bundesrepublik Deutschland hat sich ein Bewusstsein für Kinder als Träger von eigenen, einklagbaren Rechten entwickelt. Viele Nichtregierungsorganisationen fordern die Aufnahme der Kinderrechte in das Grundgesetz.

Für soziale Organisationen bedeutet dies in der Praxis, dass die politischen Entscheidungsträger auf allen Ebenen aufgefordert werden, sich weiterhin für die Rücknahme des Vorbehalts und auch für die Aufnahme von Kinderrechten in das deutsche Grundgesetz einzusetzen.

Für Flüchtlingskinder würde die Aufnahme der Kinderrechte in das Grundgesetz bedeuten, dass das Wohl auch eines Flüchtlingskindes in der Rechtshierarchie höher stünde als ausländer- und asylrechtliche Vorschriften, dass also das Wohl des Kindes vorrangig zu prüfen wäre, bei Entscheidungen zu Ausbildung, Bewegungsfreiheit, gesundheitlichen Leistungen etc. Die Ausgrenzung von Flüchtlingskindern hätte dadurch ein Ende.

Die meisten Nichtregierungsorganisationen haben Grundsatzbeschlüsse zur Umsetzung der Kinderrechte in den eigenen Diensten und Einrichtungen gefasst und mindestens einen Diskussions- und Reflexionsprozess in Gang gesetzt. Kinderrechte sollen über alle Arbeitsfelder hinweg beachtet werden. Sie sind überall dort grundlegend zu verankern, wo mit Kindern, Jugendlichen und deren Familien mittelbar und unmittelbar zusammengearbeitet wird. Auch im Arbeitsfeld Flüchtlingssozialarbeit.

\section{Wohlfahrtsverbände als Dienstleister}

Für die Flüchtlingssozialarbeit und im Arbeitsfeld Kinder- und Jugendhilfe bedeutet das teilweise große Veränderungen und Bildung neuer Schwerpunkte in den Einrichtungen und Diensten: Ziel ist es,

- die Aufmerksamkeit auf das Thema Kinderrechte zu lenken

- die in der Konvention festgeschriebenen Rechte von Kindern und Jugendlichen bekannt und deren Bedeutung bewusst zu machen

- die Verantwortungsträger für die Rechte junger Menschen zu sensibilisieren und Erwachsene darauf aufmerksam zu machen, dass sie verpflichtet sind, die Kinderrechte zu verwirklichen

- Orientierung zu bieten bei der Auseinandersetzung und Identifikation mit den Kinderrechten

- Anregungen zu geben bei der Umsetzung der Kinderrechte

- für eine Lobby-Arbeit im Sinne der Kinderrechte zu motivieren

- die Einrichtungen und Dienste der Nichtregierungsorganisationen aufzufordern, Strukturen und Prozesse im besten Interesse der Kinder und Jugendlichen zu gestalten und

- die Umsetzung der Kinderrechte zu begleiten.

In der Konsequenz bedeutet dies, einen Sensibilisierungsprozess der Mitarbeiterinnen und Mitarbeiter für die realen Möglichkeiten der Partizipation von Kindern in der eigenen Einrichtung, aber auch außerhalb in weiteren Handlungsräumen (Schule, Familie, Freizeitbereich) anzuregen.

Erfahrungen und Befragungen in anderen Arbeitsfeldern zeigen jedoch, dass die Vorstellungen über Möglichkeiten der Mitbestimmung und die tatsächliche Ausübung von Mitbestimmung sowohl bei den Kindern als auch bei den Betreuungspersonen stark divergieren. Daher ist es zunächst notwendig, herauszufinden, wo die Erzieherinnen und Erzieher, Betreuerinnen und Betreuer, Leiterinnen und Leiter der Einrichtungen Möglichkeiten der Partizipation definieren. Damit werden Bereiche festgelegt, wo den Kindern Rechte zugestanden werden sollen. Da Kinder - und noch weniger Flüchtlingskinder - ihre Rechte in der Regel nicht erkämpfen kön- nen, müssen die Erwachsenen freiwillig auf Macht verzichten und ihnen ihre Rechte zugestehen. Welche Rechte sie den Flüchtlingskindern zugestehen wollen (und welche nicht!) muss diskutiert werden.

Bei diesem Prozess ist es nachrangig, welche und wie viele Rechte die Kinder haben; wichtig ist, dass sie Rechte haben und, dass diese verbindlich garantiert und für sie nutzbar sind. Das bedeutet dann aber auch, dass diese Rechte strukturell verankert werden müssen. Sie sollten dann in Grundsätzen, dem Konzept oder einer Hausordnung schriftlich niedergelegt werden.

Wie und in welchen Strukturen können die Kinder ihre Rechte wahrnehmen? Hier bieten sich vielfältige Möglichkeiten, wie Gruppentreffen, Diskussionsforen, Beschwerdestellen, Abstimmungsverfahren, Ombudspersonen u. v. m. an. Bei Flüchtlingskindern ist auf hinreichende Verständigung, beispielsweise auf ausreichende Sprachmittlung und bildnutzende Verfahren Wert zu legen. Auch die unterschiedliche Sozialisation ist zu berücksichtigen. In kollektiven Gesellschaftsformen steht die Meinung des Individuums hinter den Bedürfnissen und Vorstellungen der Gruppe zurück und die jüngeren begegnen den Älteren mit mehr Ehrfurcht und daher auch Zurückhaltung. Mit diesen Kindern und Jugendlichen müssen gleichberechtigte und demokratische Diskussions- und Kommunikationsformen oft erst neu gelernt und geübt werden. Dabei ist seitens der Erwachsenen eine geduldige, ermutigende, unterstützende und einfühlsame Haltung die unabdingbare Voraussetzung für die Einübung von demokratischen Partizipationsformen. Voraussetzung ist aber vor allem die Bereitstellung von Freiräumen der mitverantwortlichen Selbstbestimmung, die als Recht verstanden und einforderbar sein müssen.

Dieser Prozess benötigt neben der Bereitschaft zur Haltungsänderung erhebliche personelle und zeitliche Ressourcen, die Einbindung von Kinderrechten in Praxis und Qualitätsmanagement dient jedoch zugleich dazu, das Dienstleistungsangebot zu verbessern.

\section{Wohlfahrtsverbände als Arbeitgeber}

Ein weiteres Betätigungsfeld, um Kinderflüchtlinge zu fördern, ist die Personalentwicklung. Die Bereitstellung von Arbeits- und Ausbildungsplätzen in allen verfügbaren Bereichen (Verwaltung, Pflege, Haustechnik etc.) ist daher ein wirksames und dringend gebotenes Mittel um die Teilhabe von Flüchtlingsjugendlichen zu verwirklichen. Eine stärkere Berücksichtigung der Belange von Flüchtlingen auch in der Jugendberufshilfe ist wünschenswert. Die 
Konzipierung von schulbegleitenden Fördermaßnahmen und berufsvorbereitenden bzw. Anpassungs-Qualifizierungen ist hier ebenfalls ein Mittel um Flüchtlingsjugendlichen Chancengerechtigkeit zu ermöglichen.

Dies ist durch Erleichterungen bei dem Erhalt von Arbeitserlaubnissen - seit der Veränderung der Beschäftigungsverfahrensverordnung 2007 - realisierbar geworden. Flüchtlinge, insbesondere Jugendliche erhalten seitdem weniger arbeitsrechtliche Beschränkungen selbst bei nur geduldetem Aufenthalt.

Die Implementierung der interkulturellen Öffnung der Verbände ist bei mehreren Nichtregierungsorganisationen in vielen Handlungsfeldern bereits weit vorangetrieben; interkulturelle Öffnung als Qualitätsmerkmal ist bei den meisten als Instrument der Qualitätssicherung im Qualitätsmanagement verankert und wird in vielen Gliederungen konsequent in der Personalentwicklung verfolgt. Dies bietet einzelnen erfolgreichen Flüchtlingen die Chance der beruflichen Teilhabe und führt auf dem Wege der interkulturellen Öffnung der Regelangebote wiederum zu einer erheblichen Verbesserung der Dienstleistungsangebote. Hier sind die besonderen Bedürfnisse und die Eigenheiten der Gruppe der Flüchtlinge vielleicht noch stärker als bisher, in der Personalpolitik der Verbände zu berücksichtigen.

\section{Fazit}

Der politische und innerverbandliche Einsatz für die Gleichbehandlung und individuelle Förderung von Flüchtlingskindern ist für soziale Organisationen wichtig - sowohl für die Flüchtlingskinder selbst als auch für die Qualitätsentwicklung in den Nichtregierungsorganisationen. Nicht zuletzt ist es eine Investition in die Zukunft unserer Gesellschaft.

\section{Schriften zum Migrationsrecht}

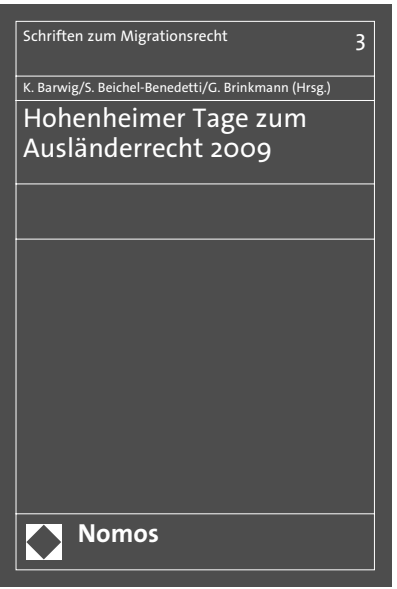

Hohenheimer Tage zum Ausländerrecht 2009

Herausgegeben von Klaus Barwig, Stephan Beichel-Benedetti und Dr. Gisbert Brinkmann 2010, Band 3, 261 S., brosch., 49, $-€$, ISBN 978-3-8329-5390-4
Der Sammelband dokumentiert die Vorträge der Hohenheimer Tage zum Ausländerrecht 2009. Die einzelnen Beiträge beschäftigen sich insbesondere mit der fortschreitenden Europäisierung des Migrationsrechts.

\author{
Der Schutz sozialer Bindungen von Ausländern \\ Eine Untersuchung zu Artikel 8 EMRK \\ Von ORR Dr. Falk Fritzsch \\ 2009, Band 2, 246 S., brosch., 56,-€, ISBN 978-3-8329-4975-4
}

Die Bedeutung des Schutzes der sozialen Bindungen (Verwurzelung) von Ausländern durch Art. 8 EMRK hat in den letzten Jahren enorm zugenommen. Der Autor erörtert umfassend das Verhältnis von EMRK und innerstaatlichem Recht und begründet die Begrenzung des Schutzbereiches auf rechtmäßige Aufenthalte.

\section{Richtlinienumsetzung: Europarechtliche Anforderungen und mitgliedstaatliche Praxis}

Eine Untersuchung am Beispiel des Einwanderungs- und Asylrechts

Von Dr. Markus Peek

2010, Band 1, 234 S., brosch., 58,- €, ISBN 978-3-8329-5041-5

Der Autor untersucht die Umsetzung migrationsrechtlicher Richtlinien und zeigt anhand konkreter Beispiele bestehende Defizite und Gestaltungsmöglichkeiten bei der nationalen Rechtsetzung auf. Ausgehend vom Konzept der Richtlinie und unter Berücksichtigung der EuGH-Rechtsprechung entwickelt er formelle und materielle Anforderungen an die Umsetzungsmaßnahmen.

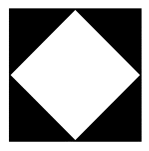

Nomos

Bitte bestellen Sie im Buchhandel oder

versandkostenfrei unter $\boldsymbol{w w w}$.nomos-shop.de 